\title{
Catheter Interruption of Atrioventricular Conduction Using Radiofrequency Energy in a Patient with Transposition of the Great Arteries
}

\author{
MARK W. RUSSELL, PARVIN C. DOROSTKAR, MACDONALD DICK, II, \\ JOSEPHA CRAENEN, * WAYNE FRANKLIN, * and BRIAN ARMSTRONG \\ From the Division of Pediatric Cardiology, The University of Michigan, C.S. Mott Children's \\ Hospital, Ann Arbor, Michigan; and *the Department of Pediatrics, The Ohio State University, \\ Children's Hospital, Columbus, Ohio
}

RUSSELL, M.W., ET AL.: Catheter Interruption of Atrioventricular Conduction Using Radiofrequency Energy in a Patient with Transposition of the Great Arteries. Percutaneous catheter mapping and radiofrequency ablation of the AV node-His bundle system (with subsequent transvenous endocardial ventricular pacing) were performed on an 18-year-old woman with transposition of the great arteries and intact ventricular septum and chronic arrhythmias 15 years following a Mustard operation. Exclusion of the $A V$ conduction tissue from the systemic venous circulation by the complex anatomy and the Mustard repair was circumvented by a retrograde approach across the aortic valve to the morphological right ventricle yielding access to the AV node-His bundle system. (PACE 1995; 18[Pt. I]:113-116)

atrioventricular conduction, Mustard operation, radiofrequency ablation, supraventricular tachycardia, transposition of the great arteries

\section{Introduction}

Postoperative supraventricular arrhythmias, including sick sinus syndrome, atrial flutter, atrial fbrillation, and other forms of atrial reentry tachycardia are common and difficult to treat complications of the Mustard operation for transposition of the great arteries. ${ }^{1-3}$ Permanent interruption of atrioventicular (AV) conduction is one definitive method that will ameliorate the symptoms associated with the rapid ventricular response that can accompany the atrial arrhythmias. ${ }^{4}$ We have previously reported the complete interruption of AV conduction using radiofrequency energy performed in the operating room on an 18-year-old

Dr. Russell is supported in part by the Kenneth M. Rosen Fellowship in Cardiac Pacing and Electrophysiology, North American Society of Pacing and Electrophysiology.

Address for reprints: Macdonald Dick, II, M.D., Box 0204 F1310, C.S. Mott Children's Hospital, University of Michigan, Ann Arbor, MI 48109-0204. Fax: (313) 936-9470.

Received February 9, 1994; revision March 25, 1994; accepted May 31, 1994. woman with chronic atrial arrhythmias following the Fontan operation. ${ }^{5}$ In this article we present a patient with complete d-transposition of the great arteries who, following the Mustard operation, developed intractable chronic atrial arrhythmias. Catheter His-bundle mapping and radiofrequency ablation were performed.

The patient, because of transposition of the great arteries and intact ventricular septum, underwent the Mustard operation at 26 months of age after a balloon atrial septostomy as a newborn. In April 1993, she experienced an in-hospital cardiac arrest due to wide QRS tachycardia; she was successfully resuscitated. In July 1993, a left ventricle to pulmonary artery conduit was placed for relief of subvalvar pulmonary stenosis; a cardioverter defibrillator pacemaker (model PCD MN7217B, Medtronic, Inc., Minneapolis, MN, USA) was also implanted. Her postoperative course was complicated by frequent atrial arrhythmias with rapid ventricular response leading to hemodynamic instability and multiple cardiac arrests. In October 1993, after multiple medications failed to control 
her tachyarrhythmias, ablation of AV conduction was proposed.

A 7 French steerable electrode catheter (4-mm tip, Mansfield-Webster, Watertown, MA, USA) was passed antegradely from the left femoral vein through the inferior vena cava to its junction at the physiological right atrium. The catheter was then passed through a small defect in the inferior limb of the Mustard baffle into the right ventricle. A sharp His-bundle potential was recorded (Fig. 1) through the two distal electrodes (interelectrode distance $=2 \mathrm{~mm}$ ). However, an accompanying atrial electrogram could not be detected, even dur-

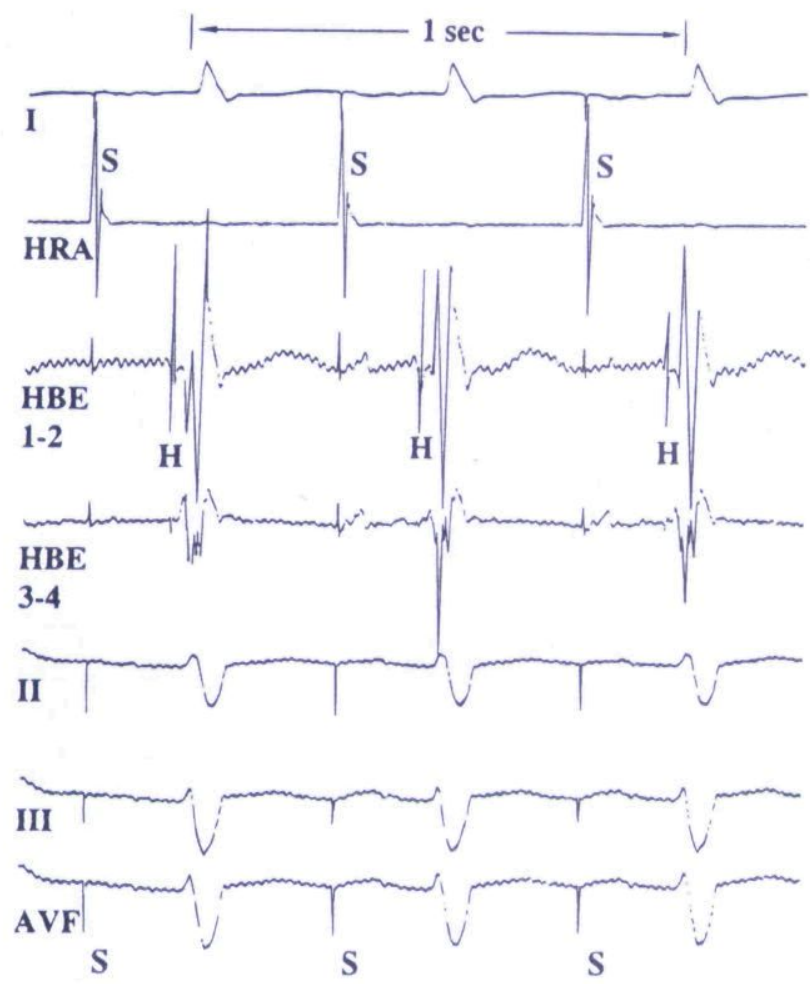

Figure 1. Recordings obtained antegrade through the defect in the Mustard baffle and demonstrates a clear narrow sharp His $(H)$ potential, short $H$-V interval, and the absence of an atrial electrogram, indicating that the distal pair of recording electrodes is both adjacent to and parallel with the His bundle, but, as suggested by the absence of an A wave, too far downstream from the $A V$ node for successful ablation. The tracings have been redrawn for clarity. (Scale of HBE tracing: $40 \mathrm{~mm} / \mathrm{mV}$.) $A V F=$ electrocardiographic lead $A V F ; H=H$ is bundle electrogram; $H B E=H i s$ bundle electrogram tracings; $I$ = electrocardiographic lead I; III = electrocardiographic lead III; $S=$ stimulus artifact. ing slow withdrawal of the catheter upstream from that site. Because of the well formed large electrogram recorded at that site, radiofrequency energy (generator RFG-3C, Radionics, Burlington, MA, USA) was delivered at 25 watts for 6 to 10 seconds in 10 unsuccessful attempts to interrupt AV conduction. A 7 French steerable electrode catheter (4-mm tip, Mansfield-Webster) was then passed retrogradely from the aorta into the systemic right ventricle. The catheter tip was manipulated across the aortic valve to the region below the crista supraventricularis and oriented posteriorly toward and over the septal leaflet of the tricuspid valve adjacent to the region of the $\mathrm{AV}$ conduction system. A smaller, broader His-bundle potential, as well as unequivocal atrial and ventricular elec-

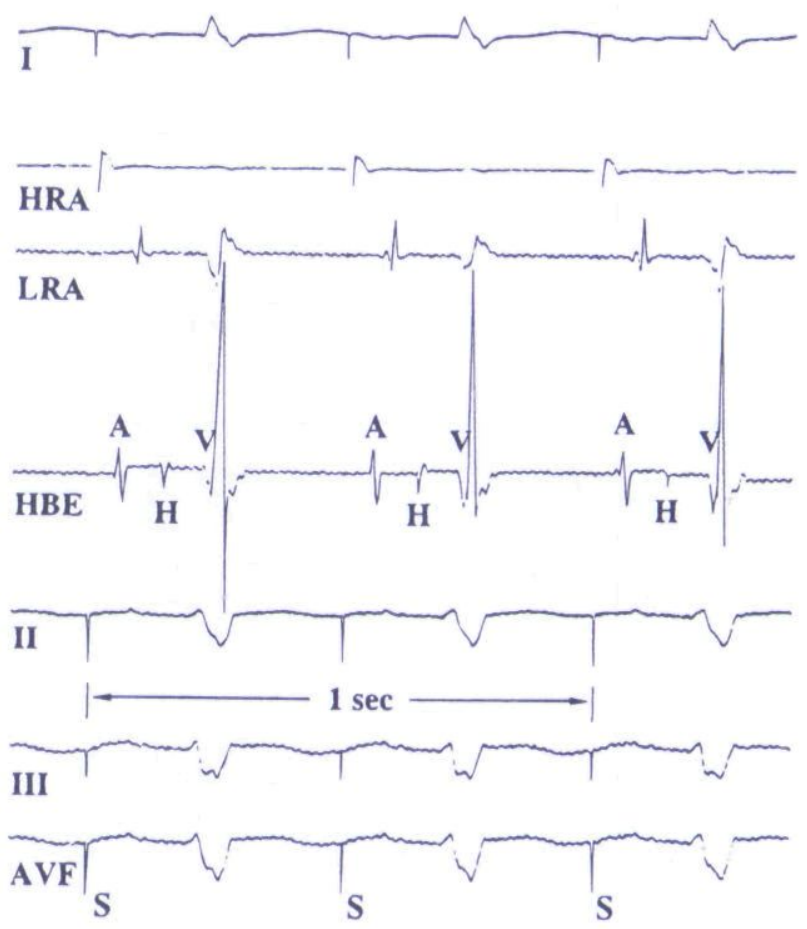

Figure 2. This figure portrays both the His $(H)$ potential, a longer $H-V$ interval, and the atrial (A) electrogram, indicating that the distal pair of electrodes is now further upstream compared to the position in Figure 1, and, therefore, the recording electrodes are closer to the atrioventricular node at this site than at the site shown in Figure 1. The tracings have been redrawn for clarity. (Scale of HBE tracing: $20 \mathrm{~mm} / \mathrm{mV}$.) $A=$ atrial electrogram; HRA = high right atrium; LRA = low right atrium; $V$ = ventricular electrogram; other abbreviations see Figure 1. 

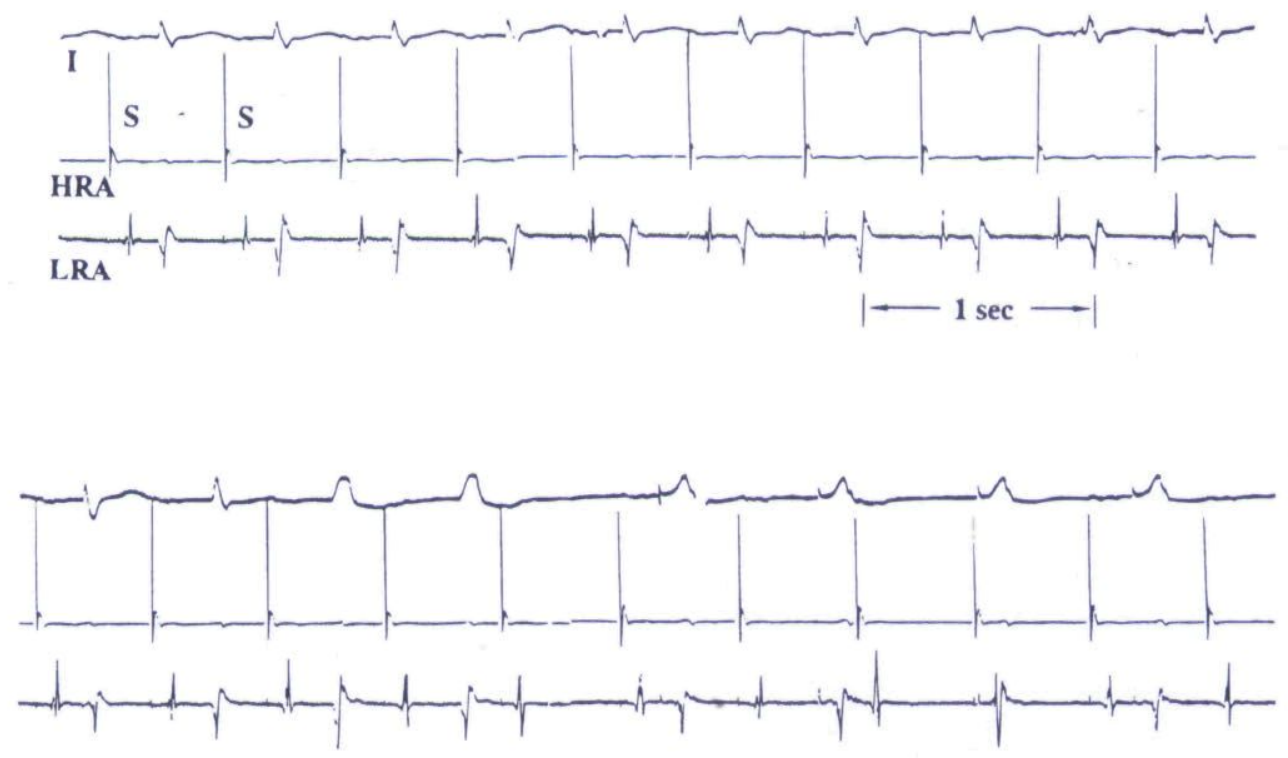

Figure 3. This tracing is recorded during delivery of radiofreqency energy (beginning at the second from the last beat on the top tracing) between an indifferent lead placed over the left scapula on the back and the distal electrode positioned at the site used to map the AV node and His bundle illustrated in Figure 2. The top and bottom tracings are continuous. Note atrioventricular block beginning with the third A electrogram in the bottom tracing, progressing to complete heart block with external ventricular pacemaker escape. The tracings have been redrawn for clarity. See Figures 1 and 2 for abbreviations.

trograms, were recorded (Fig. 2) at this site. Radiofrequency energy was delivered at this site resulting in permanent complete AV block (Fig. 3). A transvenous VVIR pacemaker was implanted with the endocardial lead in the left ventricle. The patient has continued to exhibit complete heart block and a normally functioning VVIR pacemaker.

Supraventricular tachyarrhythmias occur in up to $40 \%$ of patients following the Mustard operation for transposition of the great arteries, are often poorly tolerated in these patients and have been one of the few identified risk factors for sudden cardiac death in patients after a Mustard operation. ${ }^{1}$ This report presents the management of a patient with chronic atrial arrhythmias following a Mustard operation using percutaneous AV nodeHis bundle ablation. Although a chronic intraatrial reentry tachycardia in at least one patient following the Mustard operation for transposition of the great arteries has been successfully treated using radiofrequency energy, this patient also had atrial flutter, which was not treated with ablation. ${ }^{6}$ In contrast to that patient, the severe hemody- namic consequences (cardiac arrest) of our patient's atrial arrhythmia required definitive control. Because there is atrial-ventricular concordance in patients with complete d-transposition of the great arteries, similar to patients with normal hearts, the AV node can be found in the usual location at the apex of the triangle of Koch just posterior and inferior to the central fibrous body. ${ }^{7}$ Nonetheless, transvenous access to the AV node and His bundle in a patient following the Mustard procedure may be difficult to achieve since the AV node and His bundle are often excluded, by operative design, from the "right atrium," thus preventing not only optimal recording of equiphasic atrial, His bundle, and ventricular electrograms, but also sufficient tissue-tip contact for successful ablation. Although the retrograde aortic approach to the intraventricular His bundle and the left bundle branch of the AV conduction system has been described in patients with ventricular-arterial concordance (i.e., normal heart, the normal great vessel-ventricular relationship), ${ }^{8}$ retrograde access to the AV conduction system in a patient with ven- 
tricular-arterial discordance (i.e., d-transposition of the great arteries) would not be expected, due to the transposition, to be identical to that found in the normal heart. The subaortic conus and the insertions of the tricuspid valve chordae onto the right ventricular.septum as well as the irregular surface found in the right ventricle could interfere with precise placement and contact of the catheter electrode tip at the target conduction tissue. Yet, the curved steerable catheter could be "hooked"

\section{References}

1. Flinn CJ, Wolff GS, Dick M II, et al. Cardiac rhythm after the Mustard operation for complete transposition of the great arteries. N Engl J Med 1984; 310(25): 1635-1638.

2. Duster MC, Bink-Boelkens MTE, Wampler D, et al. Long-term follow-up of dysrhythmias following the Mustard procedure. Am Heart J 1985; 109: 1323-1326.

3. Handler CE, Graves M, Walker JM. Permanent transvenous pacing after a Mustard procedure. PACE 1989; 12:1733-1736.

4. Scheinman MM, Laks MM, DiMarco J, et al. Current role of catheter ablative procedures in patients with cardiac arrhythmias: A report for health professionals from the Subcommittee on Electrocardiography and Electrophysiology, American Heart Association. Circulation 1991; 83:2146-2153. posteriorly toward and slightly across the tricuspid valve so that the bipolar electrode tip seated at the junction of the central fibrous body and the septal leaflet of the tricuspid valve at the apex of the triangle of Koch (Fig. 2). We conclude, therefore, as postulated, ${ }^{5}$ that complete interruption of AV conduction can be achieved in a patient with complex anatomy through a transcatheter retrograde aortic to ventricular approach exploring the AV groove with the electrode tip.

5. Urcelay G, Dick M II, Bove EL, et al. Intraoperarive mapping and radiofrequency ablation of the His bundle in a patient with complex congenital heart disease and intractable atrial arrhythmias following the Fontan operation. PACE 1993; 16:1437-1440.

6. Van Hare GF, Lesh MD, Stanger P. Radiofrequency catheter ablation of supraventricular arrhythmias with congenital heart disease: Results and technical considerations. J Am Coll Cardiol 1993; 22: 883-890.

7. Wilcox BR, Anderson RH. Surgical Anatomy of the Heart. New York, NY, Raven Press, 1985, pp. 2.8-7.8.

8. Sousa J, El-Atassi R, Rosenheck S, et al. Radiofrequency ablation of the atrioventricular junction from the left ventricle. Circulation 1991; 84: $567-571$. 
This document is a scanned copy of a printed document. No warranty is given about the accuracy of the copy. Users should refer to the original published version of the material. 